\title{
Satisfacción en pacientes con reconstrucción mamaria con colgajo D.I.E.P.
}

Patient's satisfaction after diep flap reconstruction

\author{
Cabrera Sánchez, E.*, Redondo Camacho, A.**, Dean Ferrer, A.***, Benítez i Gomá, J .R.**, \\ Torre Beltrami, C.**, De Piero Belmonte, G.*, Navarro Rodríguez, J .*, Molina Sánchez, H.**at*, \\ Ramírez Sobrino, J .N.****, Rioja Torrejón, L.F.*kł***
}

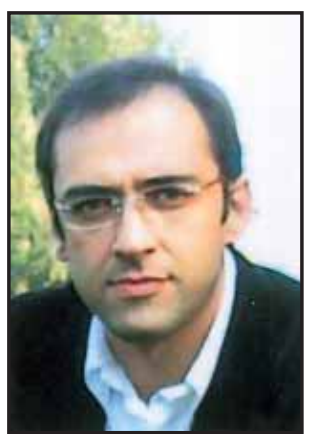

Cabrera Sánchez, E.

\section{Resumen}

La reconstrucción mamaria tras mastectomía se realiza primordialmente para proporcionar calidad de vida a la paciente. Este estudio se desarrolló para valorar la satisfacción y calidad de vida de las pacientes reconstruidas mediante colgajo D.I.E.P. (Deep Inferior Epigastric Perforator) y evaluar el resultado estético de la reconstrucción.

Treinta y tres pacientes, de 51 reconstrucciones mamarias con colgajo DIEP realizadas entre enero de 2000 y noviembre de 2004 fueron preguntadas acerca de aspectos generales relativos a la cirugía, imagen corporal y sensación subjetiva.

El resultado de imagen corporal fue evaluado por dos observadores externos, un cirujano plástico y una enfermera, además de por la propia paciente. Para valorar la reconstrucción mamaria, se utilizó una escala de 4 puntos.

La satisfacción general de nuestro estudio fue más elevada que la observada en estudios precedentes. Hemos conseguido una valoración alta en simetría, dentro de los parámetros objetivos y en integridad corporal entre los subjetivos. Hemos encontrado una correlación alta entre las respuestas de los observadores comparada con las respuestas de las propias pacientes. Por último, hemos visto una fuerte correlación entre integridad corporal y satisfacción general.

\section{Palabras clave Reconstrucción mamaria, Satisfacción, Colgajo DIEP}

Código numérico 52140, 158332
Breast reconstructions after breast cancer surgery are primarily performed to improve patient's quality of life. This study investigates patient's satisfaction and quality of life with breast reconstruction after deep inferior epigastric perforator (D.I.E.P.) flap surgery and to evaluate the aesthetic result of the breast reconstruction.

Thirty-three patiens, from fifty-one DIEP breast reconstruction made between january 2000 and december 2004 were answered about three questionnaires concerning to general aspect, body image, and subjective sensation. The body image outcome was also evaluated by one plastic surgeon and a nurse. The panel evaluated breast reconstruction on 4 subescales.

General satisfaction in our study was higher than in previous ones. We have received a high degree of symmetry in the objetive evaluation and a high score in body integrity in the subjective evaluation. We have found a stronger correlation between clinical observer answers compared with patient's assessment and a strong correlation between body integrity and general satisfaction outcome.

* Médico Interno Residente.

* Médico Adjunto de Cirugía Plástica y Reparadora.

* J Jefe de Sección de Cirugía Maxilofacial.

* Doctor en Ciencias Económicas y Empresariales. Departamento de Gestión Empresarial y Métodos Cuantitativos de la Facultad de Ciencias Económicas y Empresariales (ETEA) de la Universidad de Córdoba.

* J J efe de Servicio de Cirugía Plástica y Reparadora.

Servicio de Cirugía Plástica y Reparadora. Hospital Regional Universitario «Reina Sofía». Córdoba (España).

Trabajo presentado al Premio de Residentes del XL Congreso de la FILACP. Buenos Aires. Marzo de 2006. 


\section{Introducción}

El objetivo de toda cirugía de reconstrucción mamaria es conseguir el máximo nivel estético (forma, volumen, textura y simetría mamarias), proporcionando resultados duraderos en el tiempo y, todo ello, con la mínima morbilidad para la paciente (1).

El desarrollo y perfeccionamiento durante la pasada década de las técnicas microquirúrgicas y, en concreto, de los colgajos de perforantes, nos ha abierto un nuevo horizonte en la reconstrucción mamaria. De todos estos colgajos, sin duda, es el DIEP (Deep Inferior Epigastric Perforator), el colgajo de perforantes de primera elección para reconstrucción mamaria, por las características propias de la grasa y piel abdominales (2).

Con este tipo de reconstrucción podemos conseguir: la durabilidad en el tiempo que proporciona la reconstrucción con tejidos antólogos y un volumen de tejido suficiente, prescindiendo del uso de implantes mamarios y de sus inconvenientes asociados (3). Todo ello con una mínima morbilidad de la zona donante y el beneficio estético añadido de una abdominoplastia $(4,5)$.

Estudios previos han valorado la satisfacción de la reconstrucción mamaria bajo diferentes métodos de reconstrucción (implantes, colgajos pediculados latissimus dorsi, TRAM y TRAM libre), pero sin embargo no son muchos los que hablan acerca de la satisfacción en reconstrucción mamaria mediante colgajo DIEP.

Con este estudio tratamos de valorar la satisfacción general de las pacientes reconstruidas, de forma diferida mediante colgajo DIEP, así como aspectos más concretos acerca de parámetros objetivos en cuanto a estética mamaria y calidad de vida de las pacientes reconstruidas mediante este método.

\section{Material y Método}

El estudio se ha realizado sobre una serie de 33 casos de reconstrucción mamaria diferida con colgajo DIEP, realizados por uno de los autores del mismo, entre los años 2000 y 2004, en la Unidad de Reconstrucción Microquirúrgica del Servicio de Cirugía Plástica y Reparadora del Hospital Regional Universitario "Reina Sofía" de Córdoba. (España).

\section{Selección de pacientes}

En principio, fueron incluidas en este estudio todas las pacientes reconstruidas mediante colgajo DIEP entre enero de 2000 y diciembre de 2004 en las que se cumpliese un mínimo de 6 meses desde que se realizara la última cirugía del proceso de reconstrucción y la fecha de inicio del estudio y en las que, lógicamen-

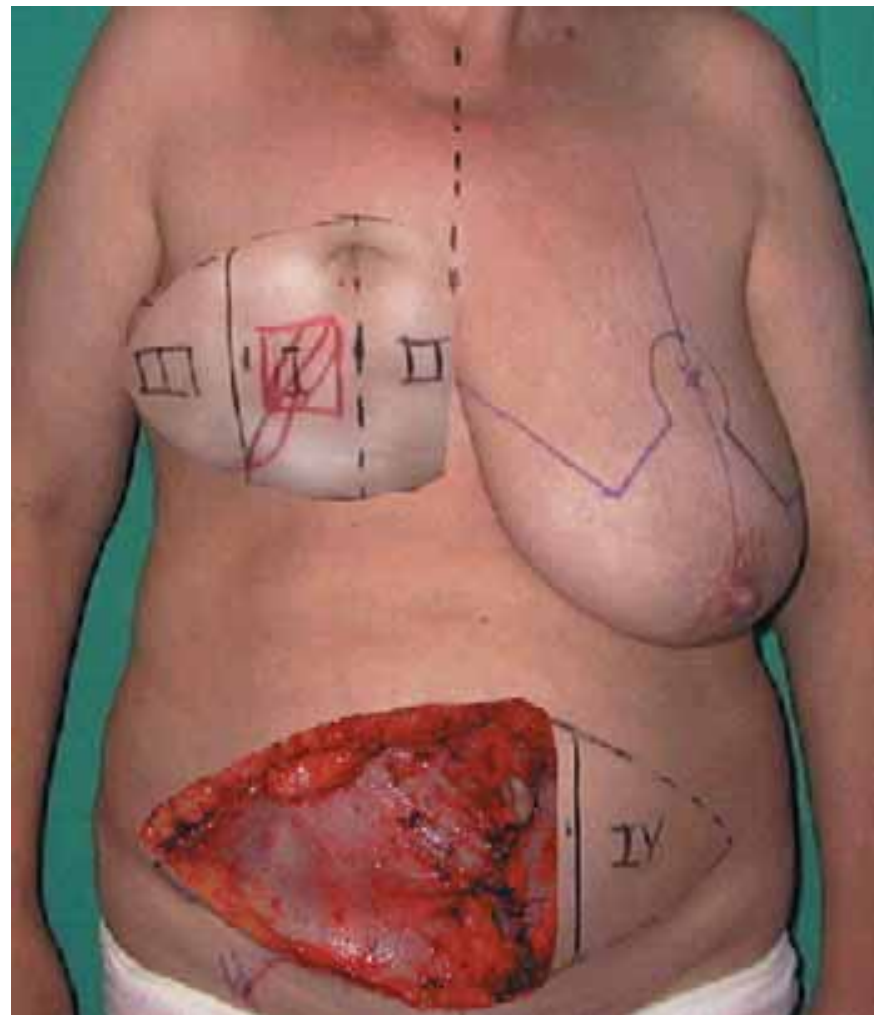

Fig. 1. Posicionamiento del colgajo. Colgajo de pedículo homolateral, se descarta la zona IV. Cicatriz umbilical de localización superior.

te no hubiese concurrido una necrosis total del colgajo: 51 pacientes en total

Las pacientes fueron llamadas telefónicamente por uno de los autores dos semanas antes de la realización del estudio y de ellas se excluyeron 18 por diferentes razones: necrosis total del colgajo (1 caso), imposibilidad de contactar telefónicamente (2 casos), CAP (Complejo Areola Pezón) no reconstruido (6 casos), causas médicas (2 casos), cuestiones geográficas o laborales (6 casos) o no asistencia el día de la valoración (1 caso).

\section{Técnica quirúrgica}

De la técnica quirúrgica en sí, no comentaremos nada ya que es conocida y se escapa del objetivo de esta publicación, pero sí de cómo realizamos el posicionamiento del colgajo (Fig. 1). Preferimos el colgajo de pedículo homolateral para conseguir una mejor adaptación y orientación del mismo, permitiendo que la zona III (dada su conformación en cuña) aporte una mayor naturalidad a la futura cola mamaria. La cicatriz umbilical, que adopta una posición superior, se desepidermiza en el proceso de remodelación y se oculta bajo el colgajo de piel torácica superior. Por último, para la recreación del nuevo surco, realizamos una resección cutánea inferior permitiendo una mejor adaptación del colgajo.

La cirugía de simetrización mamaría se realizó siempre en un segundo tiempo quirúrgico con objeto 

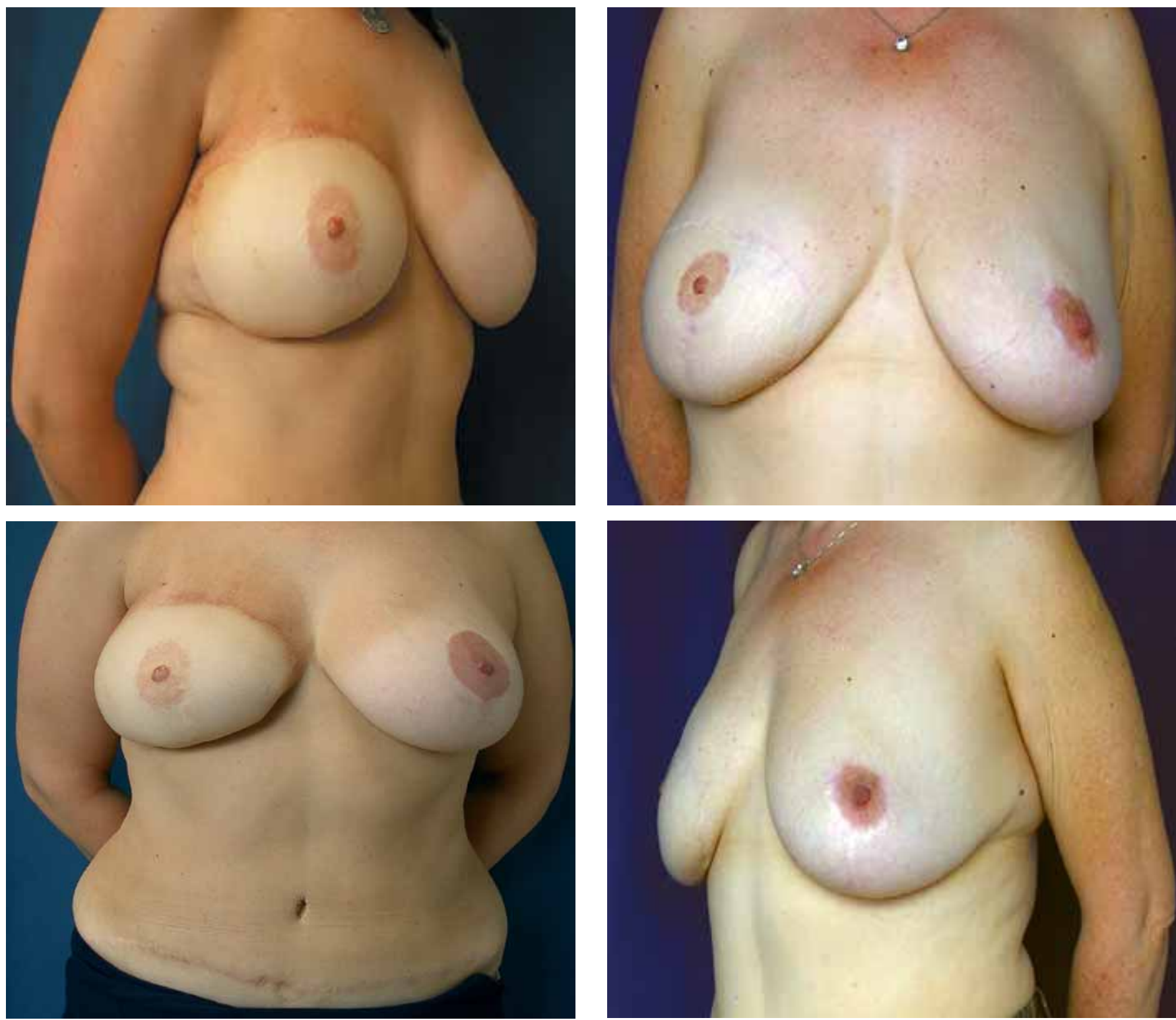

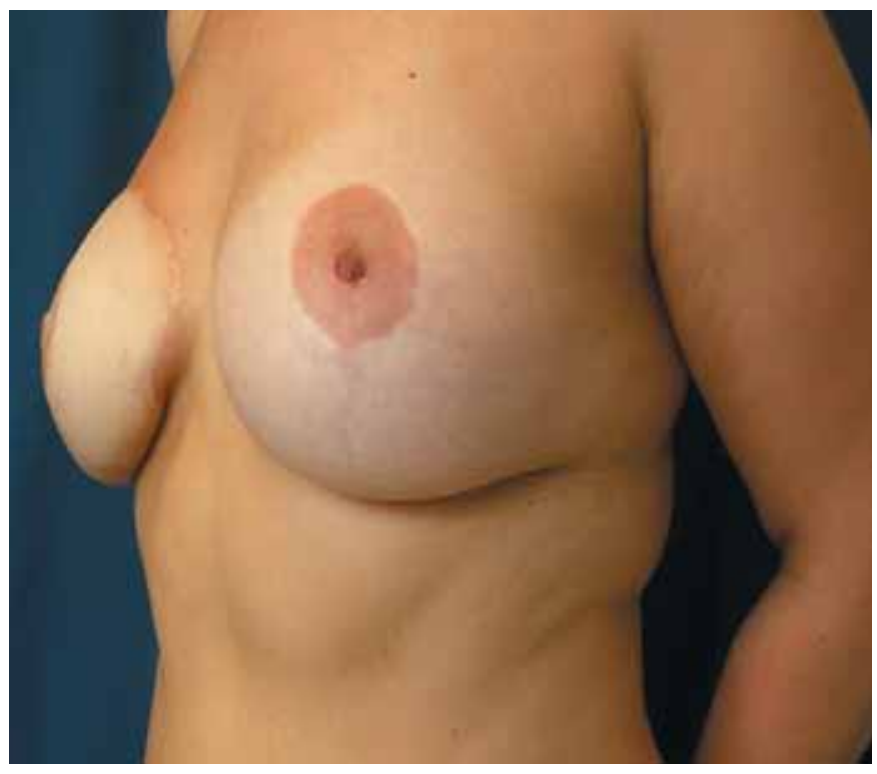

Fig. 2. Paciente de 42 años, 3 años después de reconstrucción mamaria derecha postmastectomía, con colgajo DIEP. La simetrización de la mama contralateral (mastopexia) y la reconstrucción del CAP (mediante colgajo local tipo C-V para el pezón e injerto de piel total de la región inguinal para la areola) de la mama derecha, fue realizada en un segundo tiempo, 6 meses después de la cirugía de reconstrucción.
Fig. 3. Paciente de 51 años, 4 años después de reconstrucción mamaria derecha postmastectomía, con colgajo DIEP. Se realizó mastopexia de la mama izquierda y reconstrucción del CAP derecho, un año después

de conseguir la máxima simetría, adaptando la mama contralateral al nuevo volumen y forma de la mama reconstruída. También en ese segundo tiempo realizámos la reconstrucción del CAP mediante colgajos locales para la reconstrucción del pezón e injerto cutáneo de espesor total tomado de la región inguinal para la reconstrucción de la areola.

\section{Cuestionarios}

- Datos generales: De filiación, acerca de la mastectomía y tratamientos adicionales, tiempos de reconstrucción, etc.

- Expectativas previas a la cirugía: Estética mamaria, complicaciones, dolor postoperatorio, recuperación, etc. 

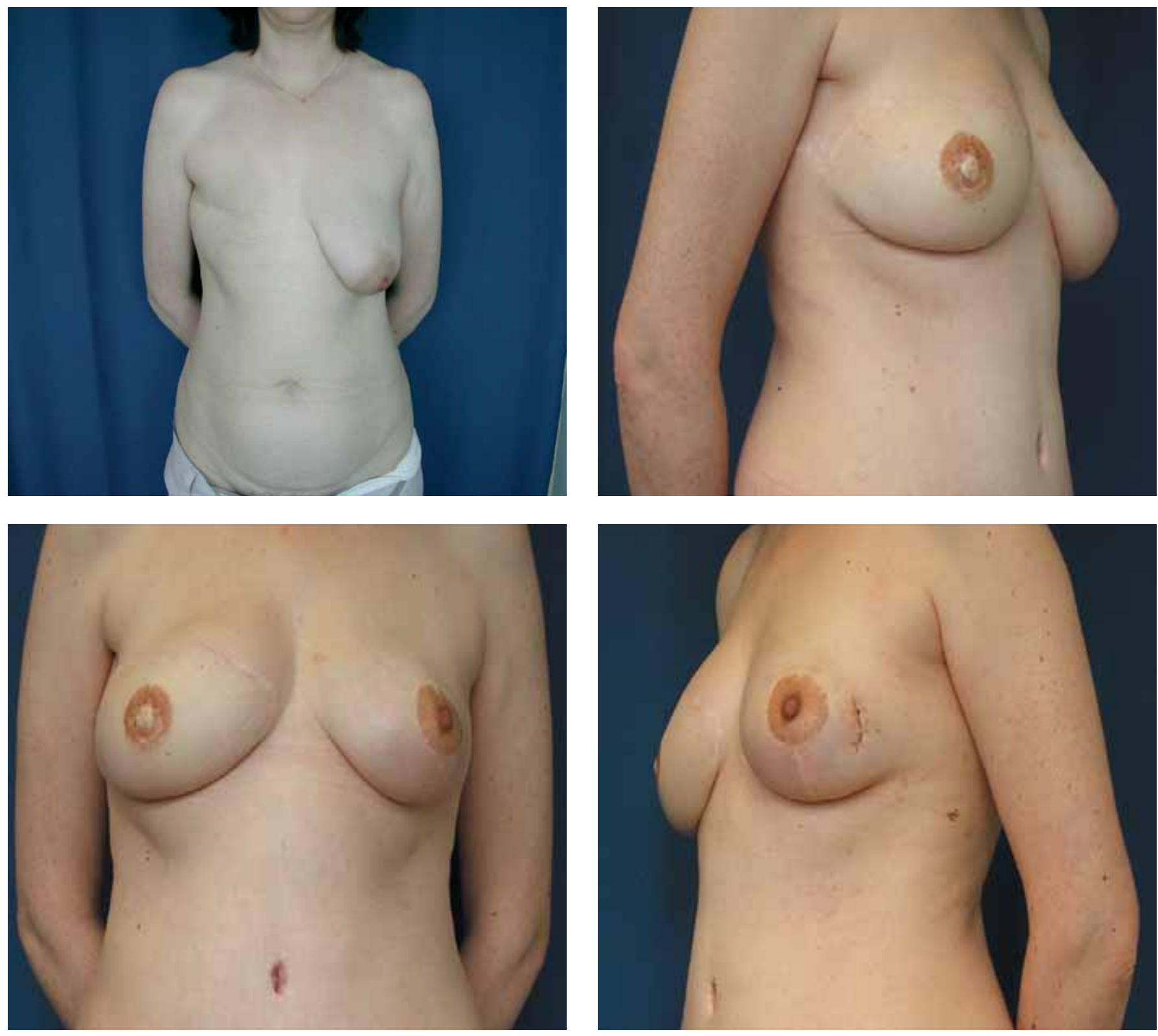

Fig. 4. Paciente de 43 años preoperatorio y dos años después de reconstrucción mamaria derecha postmastectomía, con colgajo DIEP. Se hace evidente la despigmentación parcial del CAP reconstruido (mama derecha); la cicatriz en la unión de cuadrantes externos de la mama izquierda, corresponden a una tumorectomía reciente.

- Resultados de imagen corporal: Estética mamaria, cicatrices en mama y abdomen, etc.

Este apartado relativo a imagen corporal fue evaluado, además de por la paciente, por dos observadores externos: una enfermera instrumentista de nuestro quirófano y un cirujano plástico especialista también de nuestro Servicio, pero que no participó en este estudio.

- Sensación subjetiva (satisfacción) de la enferma: basándonos en los módulos de calidad de vida en cáncer de mama editados en el año 96 por la Organización Europea para la Investigación y Tratamiento del Cáncer $(6,7)$, realizamos una serie de preguntas para valorar la sensación subjetiva y la satisfacción de las pacientes recosntruídas. Para las respuestas, utilizamos la escala numérica básica de 4 puntos, de manera que la máxima puntuación correspondía al 4 y la mínima al 1 , con dos valores intermedios $(8,9)$. No todos los datos recogidos en esta investigación han sido utilizados en esta publicación.

\section{Estudio estadístico}

Para el estudio estadístico hemos utilizado la versión $\mathrm{n}^{\circ} 13$ del paquete estadístico SPSS. Realizamos un estudio descriptivo, con Tablas de contingencia para contrastar independencia entre paciente y observadores $(\mathrm{H} 0=$ Independencia opinión $)$. Y por último, un análisis CHAID (contraste Chi-cuadrado). Para todo ello, hemos contado con la asistencia y asesoramiento del Departamento de Gestión Empresarial y Métodos Cuantitativos de la Facultad de Ciencias Económicas y Empresariales (ETEA) de la Universidad de Córdoba (España). 

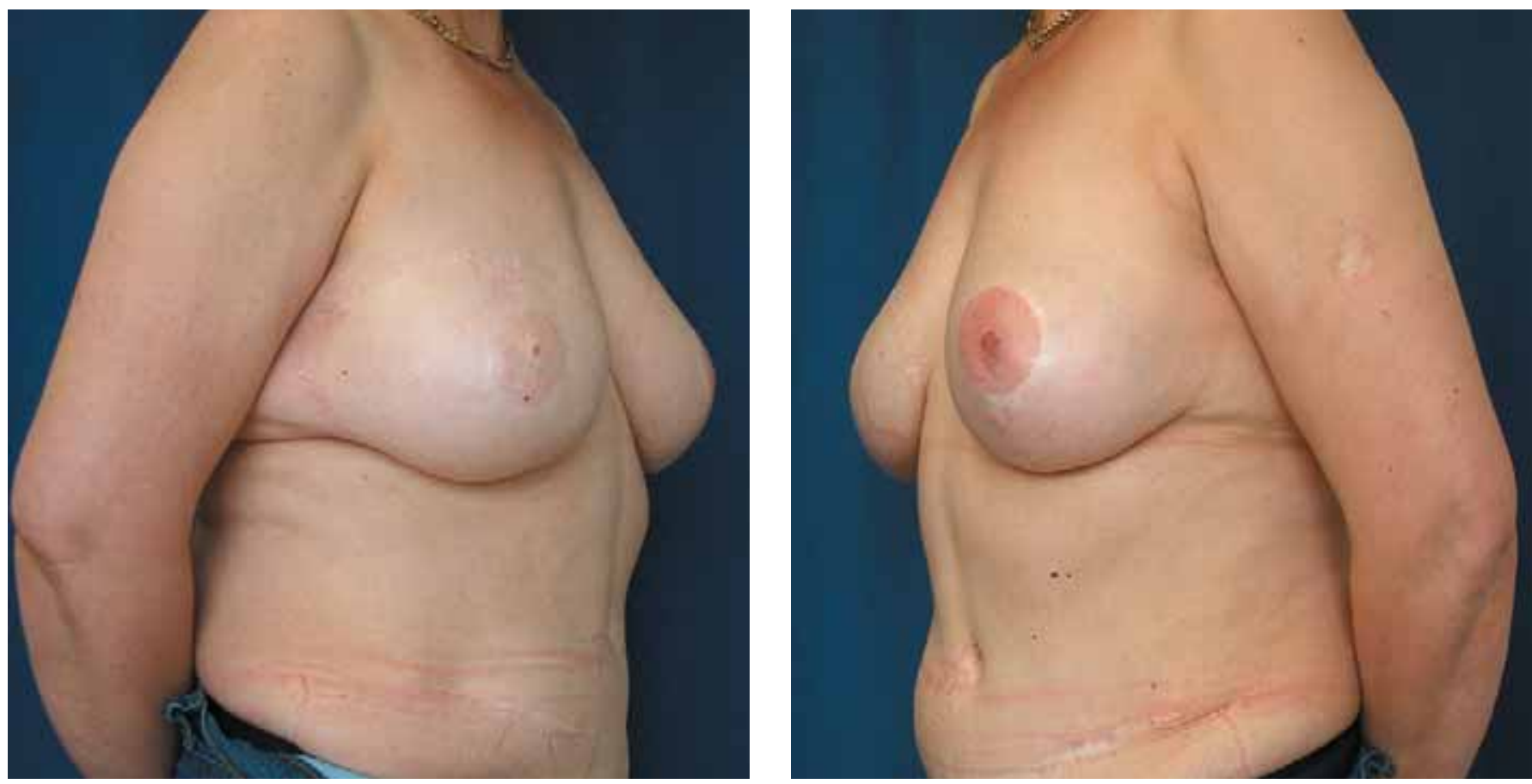

Fig. 5. Paciente de 57 años 4 años después de reconstrucción mamaria derecha postmastectomía, con colgajo DIEP. Se realizó mastopexia izquierda (cicatriz vertical) y reconstrucción del CAP derecho, que presenta despigmentación total.

Resultados

\section{Satisfacción general (Gráfico I)}

La satisfacción general de nuestro estudio fue del $93.9 \%$; es decir, que de las 33 pacientes evaluadas, tan solo dos no estuvieron satisfechas con el resultado de la reconstrucción. Estos resultados nos han impedido establecer 2 grupos (satisfechas/no satisfechas), ya que el número de casos en cada grupo era muy desigual y no comparable. Por tanto los datos que a continuación presentamos corresponden al global de los casos (33), expresados en medias.

\section{Datos Generales (Tabla I; Gráfico II)}

La media de edad de las pacientes valoradas fue de 50,71 años, con Indice de Masa Corporal (IMC) medio de 26,9, es decir, con sobrepeso. El tiempo medio transcurrido desde realizada la mastectomía a la cirugía de reconstrucción fue de 3,96 años y 1,45 años duró la totalidad del proceso reconstructivo.

Tabla I. Datos generales

\section{EDAD}

IMC

TIEMPO TRANSCURRIDO DESDE

LA MASTECTOMIA A LA CIRUGIA DE RECONSTRUCCION

TIEMPO TOTAL DEL PROCESO

DE RECONSTRUCCION MAMARIA 1,45 AÑOS
En la hoja de datos generales valoramos la percepción de las pacientes acerca del tiempo de hospitalización, de las explicaciones técnicas previas a la cirugía, del trato con el cirujano, del dolor postoperatorio y de la recuperación, obteniendo en todos estos parámetros una valoración mayor o igual a 3 , es decir un resultado bueno.

\section{Imagen Corporal (Fig. 2-5)}

Como hemos dicho anteriormente, los resultados relativos a imagen corporal fueron valorados, además de por la paciente, por dos observadores. Mediante tablas de contingencia, hemos podido ver cómo existe una fuerte consistencia en las respuestas dadas por los dos observadores, estando asociadas sus respues-

Satisfacción: 93, 9\% No satisfacción: $6,1 \%$

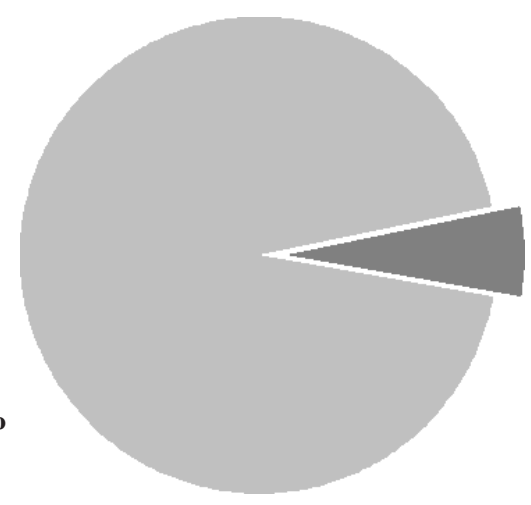

Gráfico I. Satisfacción General. De las 33 pacientes evaluadas, tan solo dos no estuvieron satisfechas con el resultado de la reconstrucción. 


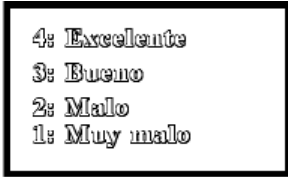

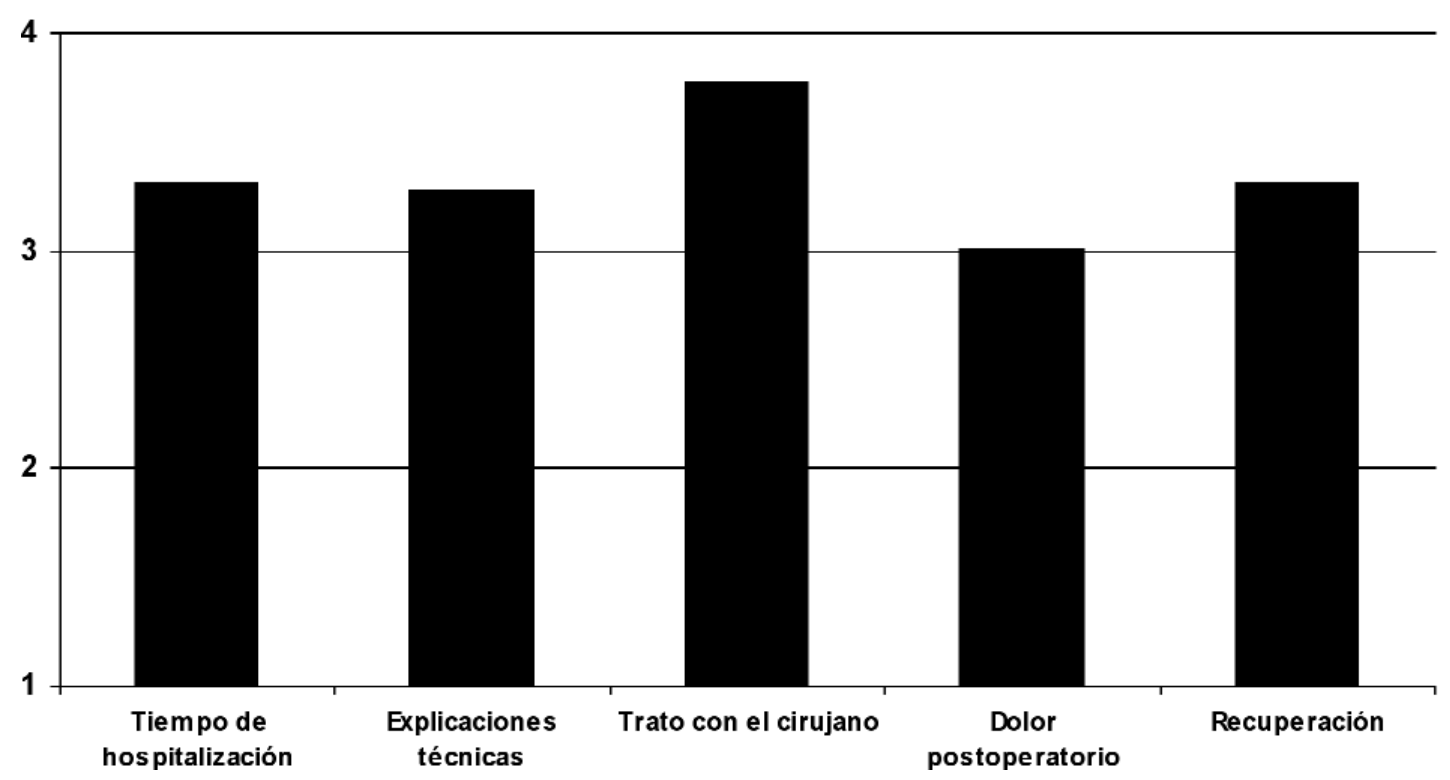

Gráfico II. Aspectos Generales. La valoración en todos los parámetros fue mayor o igual a 3, es decir un resultado bueno.

tas en todos los casos, salvo en lo relativo a simetría y color. Sin embargo, hemos comprobado cómo estas asociaciones tienen un resultado más pobre entre paciente y observadores.

La simetría mamaria, fue la valoración que obtuvo una mayor puntuación en lo relativo a imagen corporal. El 87,9 \% de las pacientes, valoraron la simetría mamaria conseguida como buena (3 pacientes) o excelente (4 pacientes). Esa misma tendencia se observó para el volumen y forma mamarias.

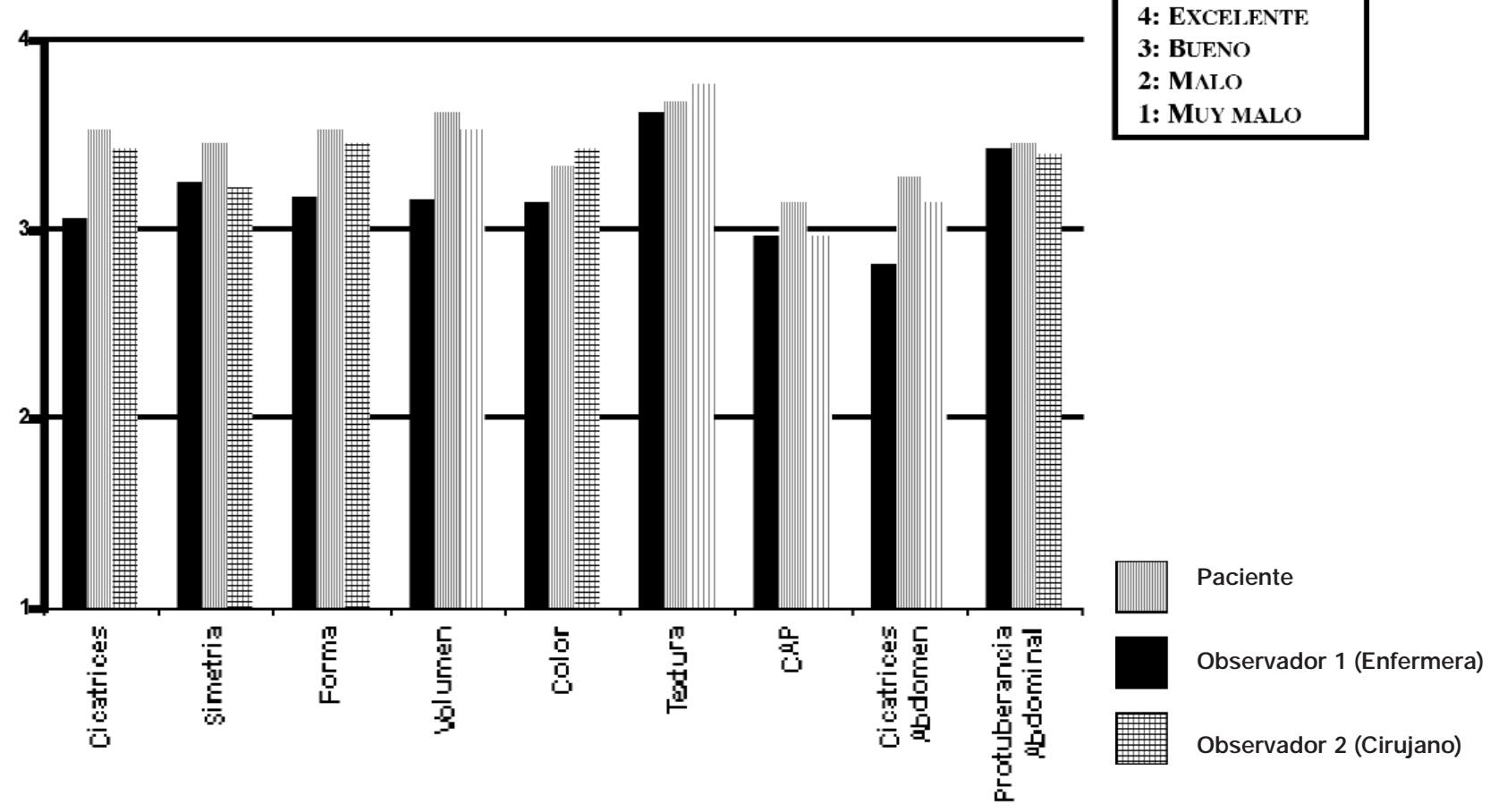

Gráfico III. Imagen Corporal. Los parámetros a los que las pacientes adjudican una mayor puntuación son simetría, forma, volumen y textura. Los peor valorados son el resultado del CAP y las cicatrices abdominales. Aunque ambos observadores coinciden en casi todos los parámetros, la concordancia es mucho menor entre pacientes y observadores. 


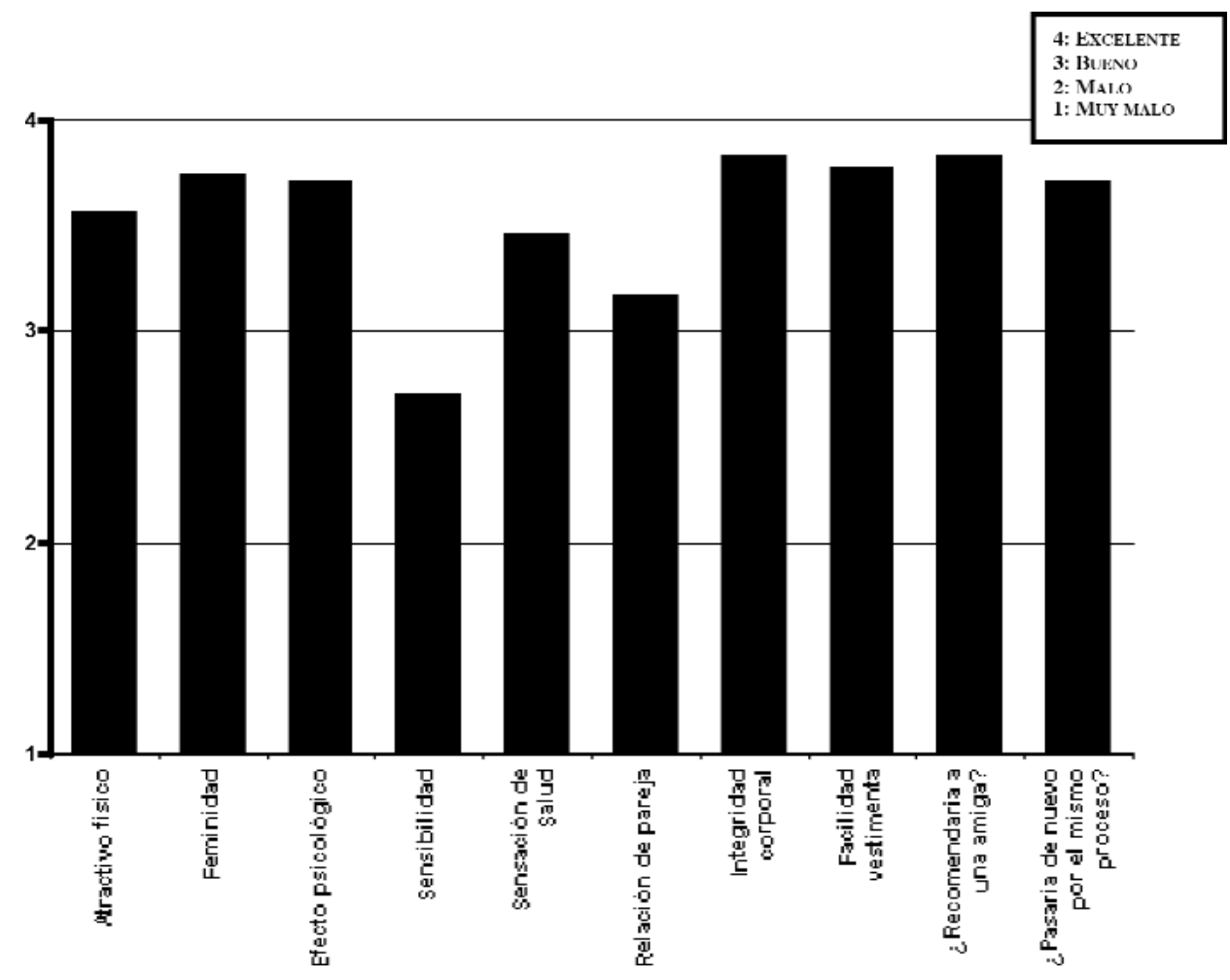

Gráfico IV. Sensación subjetiva. Las puntuaciones fueron muy altas en todos los parámetros analizados, salvo en la sensibilidad del colgajo. La integridad corporal fue el aspecto con mejor valoración, calificándola en un $81,8 \%$ de los casos como excelente.

Sin embargo, los parámetros a los que adjudicaron las pacientes una menor puntuación fueron las cicatrices en abdomen y CAP. En cicatrices en abdomen (en concreto, redundancias dérmicas laterales en el cierre de la zona donante), hay un $30,3 \%$ de pacientes que consideraron el resultado como malo (2 pacientes) o muy malo (1 paciente). De forma similar ocurre con el CAP: un $34,5 \%$ de las pacientes consideraron el resultado de la reconstrucción del CAP (baja compatibilidad de color) como malo (2 pacientes) o muy malo (1 paciente).

En la valoración del resultado del CAP y abultamiento abdominal, paciente y observadores coinciden en sus respuestas. Los resultados dados entre ambos observadores, también son muy homogéneos con respecto a estas variables.

\section{Sensación subjetiva}

Las puntuaciones fueron muy altas en todos los parámetros analizados salvo en lo referente, y como por otra parte era de esperar, a la sensibilidad del colgajo.

Dentro de estos resultados, el que dio una puntuación mayor fue el relativo a la integridad corporal, calificándola un $81,8 \%$ de casos con la máxima puntuación, es decir como excelente.

Conociendo que la integridad corporal era el parámetro de satisfacción más valorado por las pacientes, realizamos un análisis CHAID del mismo. El análisis
CHAID es una herramienta de un alto poder predictivo para ver, en qué medida, la integridad corporal influye en la satisfacción general de nuestro estudio. Con este estudio hemos conseguido detectar, con un 99,8\% de confianza, que las pacientes que valoraban en menor medida la sensación de integridad corporal (valoración $=<3$ sobre 4$)\left(\chi^{2}=9,58\right.$ con un gradote libertad, $\mathrm{p}=0,002)$ son las que menor satisfacción general tenían.

Por lo tanto podemos concluir que la sensación de integridad corporal es un parámetro importante para conseguir una elevada satisfacción de las pacientes, siendo la mejor garantía de satisfacción la obtención de una puntuación $>3$.

Como podemos observar, las dos pacientes no satisfechas presentaron una valoración $<=3$. Sin embargo, si conseguimos una puntuación $>3$ es la mejor garantía de satisfacción.

\section{Discusión}

La cirugía de reconstrucción mamaria está evolucionando hacia procedimientos que nos permiten realizar reconstrucciones de mayor calidad y durabilidad de resultados en el tiempo, con una menor morbilidad para la paciente. En este sentido, con la introducción del colgajo DIEP en la reconstrucción mamaria, el arte de este tipo de reconstrucciones ha entrado en una nueva y excitante era. 


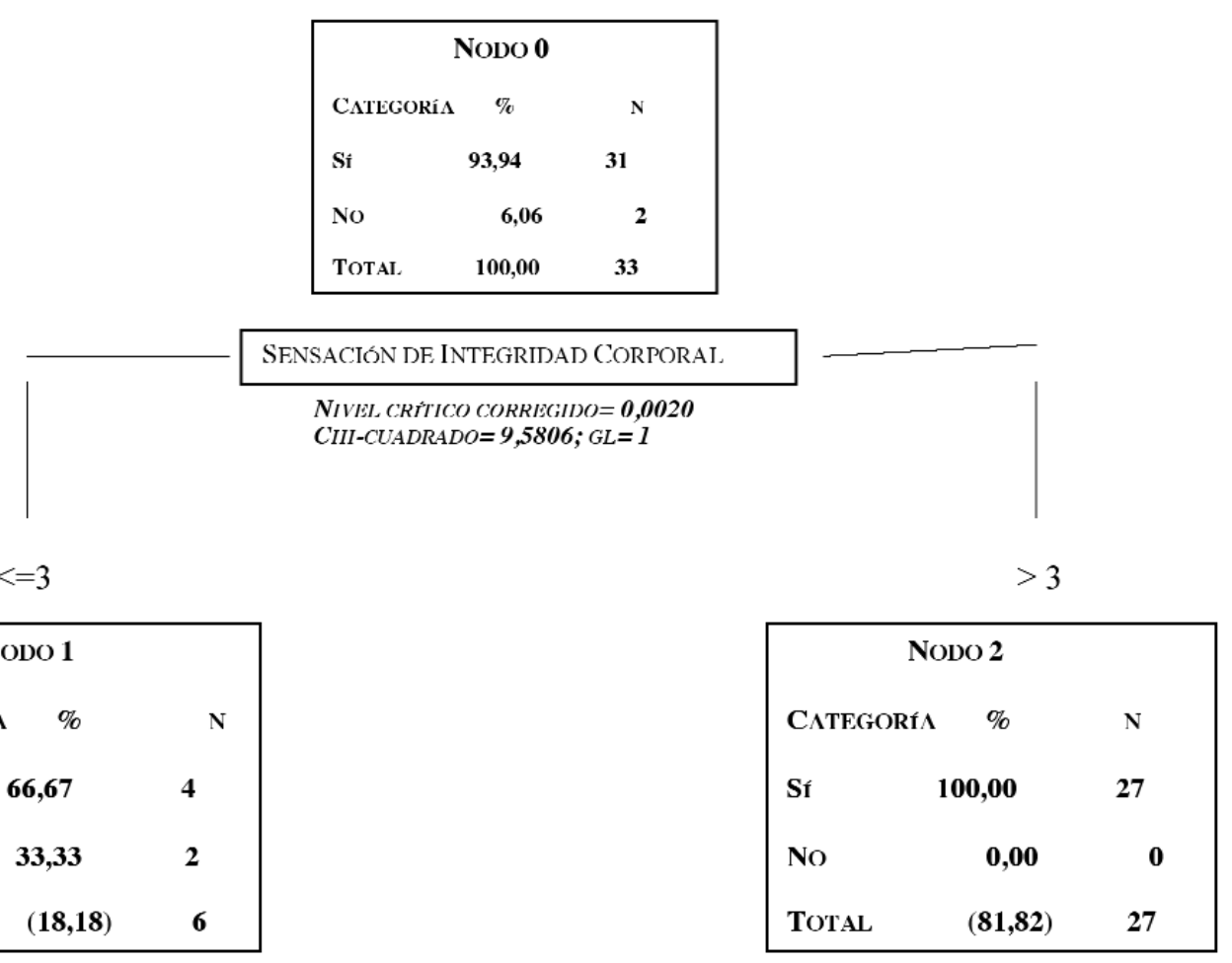

Tabla II. Estudio CH.A.I.D. Las dos pacientes no satisfechas presentaron una valoración <=3. Sin embargo, conseguir una puntuación > 3 es la mejor garantía de satisfacción.

La reconstrucción mamaria con colgajo DIEP proporciona una gran calidad en la reconstrucción mamaria, consiguiendo una mama con volumen, forma, textura y evolución en el tiempo similar al de la mama sana, junto con un beneficio estético, y mínimo sacrificio anatómico en la zona donante (4).

El estudio que aquí presentamos, corresponde a un grupo de 33 pacientes con reconstrucción mamaria mediante colgajo DIEP, operadas por el mismo cirujano en un intervalo de 4 años. La evaluación de las pacientes en términos de satisfacción objetiva, subjetiva y datos relativos a calidad de vida, se realizó transcurridos al menos 6 meses desde la finalización del proceso reconstructivo, cuando las pacientes se habían incorporado a su vida normal.

\section{Satisfacción General:}

La reconstrucción mamaria con colgajo DIEP ha sido una técnica eficaz, logrando un porcentaje de satisfacción muy elevado $(93,9 \%)$. Este porcentaje de satisfacción tan alto, junto con una muestra de estudio pequeña, nos ha impedido establecer dos grupos comparativos (satisfechas/no satisfechas), no pudiendo definir el perfil de la paciente satisfecha en relación con la no satisfecha.

El porcentaje de satisfacción obtenido en este estudio es superior al de estudios previos en los que se ha valorado la satisfacción de la reconstrucción mamaria mediante expasores o colgajos pediculados y libres (3,
$6,9,10)$. Entre los motivos que pensamos que pudieran justificar estas diferencias, podría considerarse que: nuestra muestra es más pequeña, tan solo 33 casos; que está basado en casos de reconstrucción diferida, mientras que lo publicado corresponde, en su mayoría, a reconstrucciones inmediatas; en este sentido, la reconstrucción diferida parece aportar una ligera ventaja en la satisfacción de las enfermas, ya que éstas durante un tiempo se ven sin mama, valorando más el resultado final (11-12). Por último, a que el estudio lo hemos realizado a un período (2000-2004) en el que el nivel de entrenamiento de nuestro equipo quirúrgico con este tipo de cirugía era muy elevado.

\section{Imagen Corporal:}

De forma similar a lo publicado por otros autores en reconstrucción mamaria con colgajos libres (9), hemos conseguido una valoración muy alta en simetría, forma y volumen mamario, que son la guía de este tipo de reconstrucciones. El aspecto de imagen corporal más valorado por las pacientes fue la simetría mamaria; en este sentido, pensamos que con la realización de la cirugía de simetrización de forma diferida se puede conseguir una mayor simetría que si se realiza en el mismo tiempo quirúrgico de la reconstrucción. De forma diferida, adaptamos la mama contralateral a la nueva neomama y no a un colgajo en fase aguda, que puede tener problemas de viabilidad (que alteren su forma y su volumen), y ser difícil pre- 
decir su adaptabilidad y caída sobre la pared torácica $(12,13)$.

La reconstrucción del CAP no han recibido mucha atención en la literatura en comparación con otros aspectos de la reconstrucción mamaria; sin embargo, está demostrado que es un factor muy importante en la satisfacción final de las pacientes reconstruidas (1418).

Un $34,5 \%$ de las mujeres de nuestro estudio consideran el resultado de la reconstrucción del CAP como malo o muy malo. Además, no solo las pacientes valoran de forma negativa este resultado, sino que ambos observadores hacen la misma apreciación. Por tanto, debemos hacer más hincapié en detalles de compatibilidad de color del CAP y tratamiento de cicatrices, detalles que, hasta ahora, habíamos considerado como menores, pero que una vez superadas las dificultades mayores de viabilidad del colgajo y estética mamaria, debemos tener en mayor consideración con objeto de alcanzar el máximo nivel de refinamiento en este tipo de reconstrucciones.

\section{Sensación Subjetiva:}

Para la valoración de la sensación subjetiva y calidad de vida, diseñamos un cuestionario de 10 preguntas $(6,7,10)$, obteniendo en todas ellas una valoración muy alta. Las pacientes reconstruídas reconocen sentirse más femeninas y con un mayor atractivo físico. El parámetro más valorado por todas ellas es la sensación de integridad corporal, que un $81,8 \%$ de las pacientes consideran como excelente. Gracias al análisis CHAID, hemos podido comprobar en qué medida la integridad corporal influye en la satisfacción general de las pacientes, de manera que las pacientes que valoraban en menor medida la sensación de integridad corporal son las que menor satisfacción general tenían.

Cuando les preguntábamos acerca de si recomendarían este tipo de intervención a una familiar o amiga o incluso si ellas mismas pasarían de nuevo por este proceso, hubo una gran unanimidad de respuestas, otorgando la máxima puntuación en más del $80 \%$ de los casos.

Por último, nuestro cuestionario terminaba preguntando a las pacientes que expusieran con una frase lo que la reconstrucción había supuesto para ellas (19). Pues bien, integridad corporal y recuperación de la autoestima fueron, con diferencia, las frases más repetidas por todas. Por tanto, no podemos devolver la mama perdida, ni borrar las cicatrices, pero sí conseguir un volumen, forma y textura similares a los de la mama amputada y, en definitiva, contribuir a recuperar la integridad corporal y autoestima de estas mujeres.
Conclusiones

En conclusión, los resultados obtenidos en este estudio demuestran un nivel de satisfacción general muy elevado, con una valoración muy alta en simetría y forma mamaria. Sin embargo, se hace necesario hacer estudios más consistentes en cuanto al número de casos, para ver si esa tendencia se mantiene.

\section{Dirección del autor}

\author{
Dr. Emilio Cabrera Sánchez \\ Avda. de América, 27 - 2 ${ }^{\circ}, 1^{\mathrm{a}}$ \\ 14011 Córdoba (España) \\ e-mail: iocabrera@hotmail.com
}

\section{Bibliografía}

1. Shaw WW. "Microvascular free flap breast recosntruction". Clin Plast Surg 1984, 11: 333.

2. Allen RJ, Treece P. "Deep inferior epigastric perforator for breast reconstruction". Ann Plast Surg 1994, 32:32.

3. Fee-Fulkerson, K., Conaway, M.R., Winer, E.P. "Factors Contributing to Patient Satisfaction with Breast Reconstruction Using Silicone Gel Implants". Plast and Recosntr Surg, 1996; 97 (4) : 1420.

4. Nahabedian MY, Momen B, Galdino G, Manson PN. "Breast Reconstruction with the free TRAM or DIEP flap: patient selection, choice of flap, and outcome". Plast Reconstr Surg. 2002; 110(2): 466; discussion 476.

5. Vesely J. Stupka I, Drazan L, Holusa P, Licata P, Corradini B. "DIEP flap breast reconstruction -new experience"-. Acta Chir Plast, 2001; 43(1):3.

6. Edsander-Nord, A., Brandberg, I., Wickman, M, "Quality of life, Patients' Satisfaction, and Aesthetic Outcome after Pedicled or Free TRAM Flap Breast Surgery”. Plast Reconst Surg, 2001 15:1142.

7. Tsouskas, L.I., and Fentiman, I.S. "Breast compliance: A new method for evaluation of cosmetic outcome after conservative treatment of early breast cancer". Breast Cancer Res. Treat. 1990, 15: 185.

8. Mirjam A. G. Sprangers, et al. «The European Organization for Research and Treatment of Cancer Breast Cancer-Specific Quality of Life Questionnare Module : First Results From a Three-Country Field Study". Journal of Clinical Oncology, 1996, 14 (10):2756

9. Alderman, A.K., Wilkins, E.G., Lorrey, J C., Davis, J.A. "Determinant of Patient Satisfaction in Postmastectomy Breast Reconstruction. Plast Reconst" Surg, 2000, 106 (4): 769.

10. Andrade, W.N., Baxter, N., Semple, J.L.: "Clinical Determinants of Patient Satisfaction with Breast Reconstruction". Plast Recons Surg; 2001; 107(1):46.

11. Tsouskas, L.I., and Fentiman, I.S. "Breast compliance: A new method for evaluation of cosmetic outcome after conservative treatment of early breast cancer". Breast Cancer Res. Treat. 1990, 15: 185.

12. Berry, M.G., Al-Mutfi, R.A.M., Jekinson, A.D., et al. "An audit of outcome including patient satisfaction with immediate breast reconstruction performed by breast reconstruction perfomed by breast surgeons". Ann. R. Coll. Surg. Engl. 1999; 80: 173. (Abstract).

13. Van Limbergen, E., van der Schueren, E., and Van Congelen, $K$. "Cosmetic evaluation of breast conserving treatment for mammary cancer: proposal of quantitative scoring system." Radiother. Oncol. 1989, 16. 159.

14. Jabor, M.A., Shayani, P., Collins, Jr. D.R., Karas, T., Cohen, B.F.: "Nipple-Areola Reconstruction: Satisfaction and Clinical Determinants". Plast Reconst Surg, 2002; 110(2):457

15. Wellisch, D.K., Schain, W.S., Noone, R.B., and Little, J.W., III. "The psychological contribution of nipple addition in breast reconstruction". Plast Reconst Surg 1987, 80:699.

16. Asplund, O. "Nipple and areola reconstruction: A study of 79 mastectomized women". Scand. J. Plast. Reconstr. Surg. 1983, 17:233. 
17. Kroll, S.S. "Nipple reconstruction with double-opposing tab flap". Plast Recons Surg 1999, 104:511.

18. Batí, M.A. and Berry, RB. "Nipple-areola reconstruction by tattooing and nipple sharing". Br. J. Plast Surg. 1997, 50: 331.
19. Van Dam, FS and Bergman, RB. "Phycosocial and Surgical aspects of breast reconstruction." Eur Journ Surg. Oncol.1998, $14: 141$. 